\title{
Hematoma epidural secundario al empleo de cabezal autoestático en un adulto
}

\author{
R. Serramito-García; A. Arcos-Algaba; J.M. Santín-Amo; A. García-Allut; F.J. Bandín-Diéguez y M. Gelabert- \\ González \\ Servicio de Neurocirugía. Hospital Clínico Universitario de Santiago de Compostela. Departamento de Cirugía. Universidad de Santiago de \\ Compostela.
}

\begin{abstract}
Resumen
El cabezal autoestático es un instrumento utilizado habitualmente en las intervenciones neuroquirúrgicas y a pesar de su utilización adecuada, puede ser el causante de algunas complicaciones graves como la perforación craneal y el desarrollo de hemorragias intracraneales. Presentamos el caso de un paciente varón de 19 años sometido a una ventriculocisternostomía endoscópica y que presentó un hematoma epidural secundario a la penetración intracraneal de uno de los pinchos del cabezal.
\end{abstract}

PALABRAS CLAVE: Complicación. Cabezal autoestático. Hematoma epidural. Ventriculostomía endoscópica.

Epidural haematoma due to an headrest in an adult

Summary

A head fixation device with pins is commonly used for immobilization of the patients during neurosurgical procedures. Despite its appropiate management, may be the cause of some serious complications such as skull perforation and intracranial injuries. We report the case of a 19-years-old young admitted for a endoscopic third ventriculostomy who developed an epidural haematoma due to the penetration of the skull by a pin.

KEY WORDS: Complication. Endoscopic third ventriculostomy. Epidural haematoma. Headrest.

\section{Introducción}

Los cabezales autoestáticos (de pinchos) constituyen una herramienta fundamental en las intervenciones neuroquirúrgicas craneales al permitir una fijación estricta del cráneo del paciente. Su empleo en general está reservado a pacientes mayores de 2 años, edad en la que el cráneo tiene

Recibido: 12-07-09. Aceptado: 7-10-09 un grosor aceptable para evitar la penetración intracraneal de alguno de los pinchos de fijación. Sin embargo, a pesar de su correcto empleo existen una serie de complicaciones descritas en la literatura tanto en niños como en adultos, entre las que se incluyen infecciones en el punto de inserción, desplazamiento durante la cirugía, embolismo aéreo desde la herida del pincho ${ }^{2}$, lesión vascular cutánea con hemorragia o formación de un pseudoaneurisma cutáneo por punción de un vaso del cuero cabelludo ${ }^{6}$ o penetración intracraneal del pincho sobre todo en la población infantil $^{12}$.

Presentamos el caso de un paciente adulto que fue sometido a una ventriculostomía endoscópica y que desarrolló en el postoperatorio inmediato un hematoma epidural derivado de la penetración intracraneal de uno de los pinchos del cabezal.

\section{Caso clínico}

Paciente varón de 19 años remitido desde un hospital comarcal para intervención quirúrgica por hidrocefalia. Presentaba una historia de aproximadamente 1 año de evolución de cefalea hemicraneal izquierda que empeoraba con los esfuerzos. En las últimas semanas la cefalea se incrementó en intensidad y frecuencia. La exploración neurológica mostraba únicamente un edema de papila bilateral.

El paciente aporta una resonancia magnética (RM) que muestra una dilatación ventricular supratentorial con índice de Evans de 0.43, aclaramiento periependimario en astas frontales y una pequeña lesión tectal que no se modificaba con contraste. El cuarto ventrículo era normal (Figura 1).

Con el diagnóstico de estenosis del acueducto de Silvio se realizó a través de un agujero de trépano frontal derecho, una ventriculocisternostomía premamilar endoscópica según la técnica habtiual ${ }^{3}$. Para la inmovilización de la cabeza se empleó un cabezal de Mayfield (Ohio Medical Instrument Co, Inc., Cincinnati, Oh, USA). El postoperatorio inmediato transcurre sin incidencias aunque el paciente aquejaba cefalea. A las 48 horas de la cirugía y previa al alta hospitalaria programada se realizó una nueva 


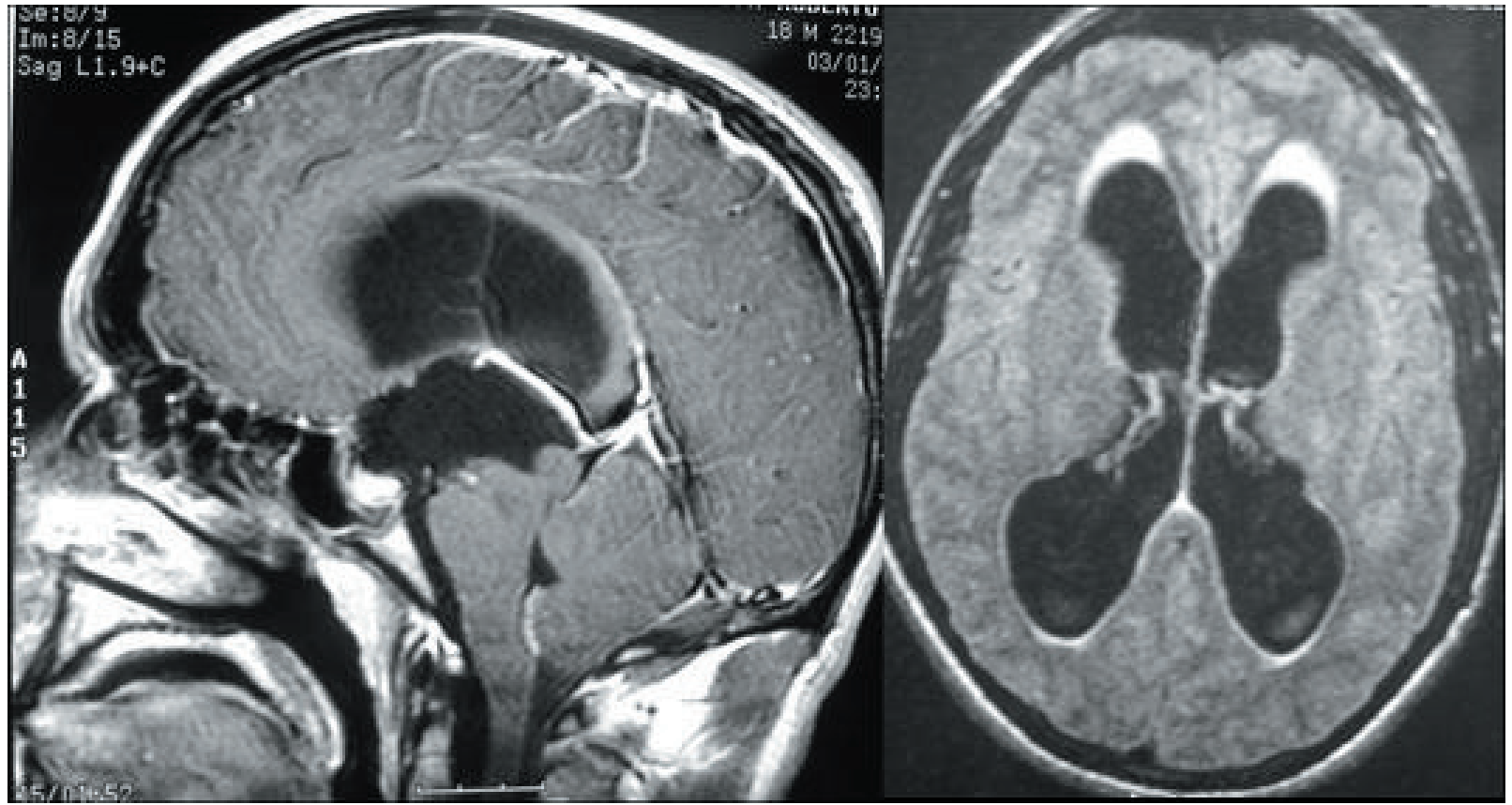

Figura 1. RM preoperatoria mostrando la dilatación ventricular supratentorial.

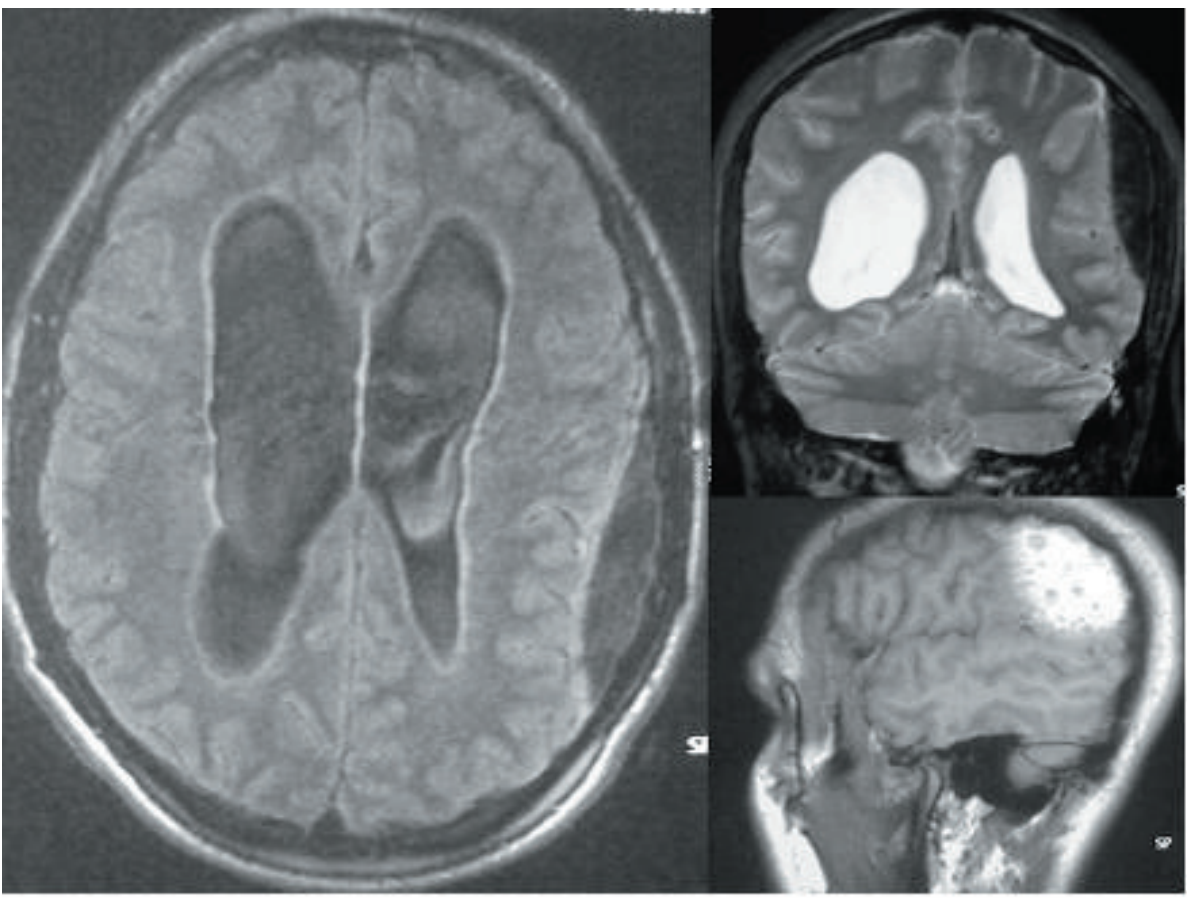

Figura 2. RM postventriculostomía: hematoma epidural parietal izquierdo con moderado efecto de masa sobre el ventrículo lateral.
RM en la que llama la atención la presencia de un hematoma epidural de localización parietal izquierda con efecto de masa sobre el ventrículo lateral ipsilateral (Figura 2).

Seguidamente se practicó una craneotomía parietal izquierda, observándose como existía una perforación que atravesaba la totalidad del hueso sin afectar a la duramadre. Se procedió al drenaje del hematoma y a la coagulación de una pequeña arteria dural sangrante. El paciente fue dado de alta a los 6 días aquejando únicamente cierto grado de cefalea. $\mathrm{Al}$ año de la cirugía se encuentra asintomático y la lesión tectal no muestra cambios en los estudios de RM.

\section{Discusión}

Los cabezales autoestáticos de inmovilización craneal constituyen una herramienta imprescindible en la práctica 
Tabla 1

Sumario de casos publicados con complicaciones derivadas del empleo del cabezal autoestático

\begin{tabular}{|c|c|c|c|c|c|c|c|}
\hline Caso & $\begin{array}{l}\text { Edad } \\
\text { Sexo }\end{array}$ & Diagnóstico & HIC & Complicación & $\begin{array}{l}\text { Momento del } \\
\text { diagnóstico }\end{array}$ & $\begin{array}{l}\text { Signo/síntoma } \\
\text { de alarma }\end{array}$ & Tratamiento \\
\hline $\begin{array}{l}\text { Cabezudo } \\
\text { et } \mathrm{al}^{2}\end{array}$ & $27 / \mathrm{V}$ & $\begin{array}{l}\text { Hemangio- } \\
\text { blastoma } \\
\text { de cerebelo }\end{array}$ & $?$ & $\begin{array}{l}\text { Embolismo } \\
\text { aéreo }\end{array}$ & Intraoperatorio & $\begin{array}{l}\text { Registro } \\
\text { con Doppler }\end{array}$ & $\begin{array}{l}\text { Sutura herida } \\
\text { cutánea y ex- } \\
\text { tracción del aire }\end{array}$ \\
\hline $\begin{array}{l}\text { De Lange } \\
\text { et } \mathrm{al}^{4}\end{array}$ & $77 / \mathrm{V}$ & $\begin{array}{l}\text { Metástasis. } \\
\text { parieto- } \\
\text { occipital }\end{array}$ & $\mathrm{Si}$ & $\begin{array}{l}\text { Embolismo } \\
\text { aéreo }\end{array}$ & Intraoperatorio & $\begin{array}{l}\text { Registro } \\
\text { con Doppler }\end{array}$ & $\begin{array}{l}\text { Sutura herida } \\
\text { cutánea }\end{array}$ \\
\hline $\begin{array}{l}\text { Fernández } \\
\text { et } \mathrm{al}^{6}\end{array}$ & $51 / \mathrm{V}$ & $\begin{array}{l}\text { Aneurisma } \\
\text { ACoA }\end{array}$ & No & $\begin{array}{l}\text { Aneurisma } \\
\text { arteria } \\
\text { temporal } \\
\text { superficial }\end{array}$ & 5 semanas & $\begin{array}{l}\text { Masa pulsátil } \\
\text { en cuero } \\
\text { cabelludo }\end{array}$ & $\begin{array}{l}\text { Resección } \\
\text { quirúrgica }\end{array}$ \\
\hline $\begin{array}{l}\text { Medina } \\
\text { et } \mathrm{al}^{9}\end{array}$ & $13 / \mathrm{V}$ & $\begin{array}{l}\text { Quiste } \\
\text { supratentorial }\end{array}$ & $\mathrm{Si}$ & $\begin{array}{l}\text { Hematoma } \\
\text { epidural }\end{array}$ & Intraoperatorio & Edema cerebral & $\begin{array}{l}\text { Craneotomía } \\
\text { y evacuación } \\
\text { del hematoma }\end{array}$ \\
\hline $\begin{array}{l}\text { Erbayraktar } \\
\text { et } \mathrm{al}^{5}\end{array}$ & $23 / \mathrm{V}$ & $\begin{array}{l}\text { Adenoma } \\
\text { hipófisis }\end{array}$ & No & $\begin{array}{l}\text { Fractura } \\
\text { craneal }+ \\
\text { epidural }\end{array}$ & Intraoperatorio & $\begin{array}{l}\text { Edema cerebral } \\
\text { e hipertensión } \\
\text { intracraneal }\end{array}$ & $\begin{array}{l}\text { Craneotomía } \\
\text { y evacuación } \\
\text { del epidural }\end{array}$ \\
\hline $\begin{array}{l}\text { Sade y } \\
\text { Mohr }^{10}\end{array}$ & $24 / \mathrm{V}$ & $\begin{array}{l}\text { Meningioma } \\
\text { supratentorial }\end{array}$ & $\mathrm{Si}$ & $\begin{array}{l}\text { Fractura } \\
\text { deprimida }+ \\
\text { epidural }\end{array}$ & 6 horas & $\begin{array}{l}\text { Crisis. } \\
\text { Derterioro } \\
\text { neurológico }\end{array}$ & $\begin{array}{l}\text { Craneotomía } \\
\text { y evacuación } \\
\text { del epidural }\end{array}$ \\
\hline $\operatorname{Yan}^{13}$ & $15 / \mathrm{V}$ & $\begin{array}{l}\text { Plexopapiloma } \\
\text { de IV ventrículo }\end{array}$ & $\mathrm{Si}$ & $\begin{array}{l}\text { Fractura } \\
\text { deprimida }+ \\
\text { hematoma } \\
\text { epidural }\end{array}$ & 6 horas & $\begin{array}{l}\text { Hematoma } \\
\text { epidural }\end{array}$ & $\begin{array}{l}\text { Craneotomía y } \\
\text { evacuación del } \\
\text { epidural }\end{array}$ \\
\hline
\end{tabular}

HIC: hipertensión intracraneal

neuroquirúrgica actual. Su empleo permite una inmovilización absoluta de la cabeza y evita las complicaciones cutáneas (escaras) y oculares que se producían cuando la cabeza se apoyaba durante largos períodos de tiempo sobre un cabezal de herradura.

Las complicaciones más graves descritas incluyen la fractura hundimiento del cráneo, la perforación craneal con lesión sobre la duramadre y cerebro y están ceñidas casi siempre a pacientes infantiles, siendo excepcionales en adultos ${ }^{10}$. Aunque existen pinchos de cabezal de pequeño tamaño, específicos para los pacientes pediátricos, y algunas ayudas técnicas para minimizar su efecto sobre el cráneo infantil ${ }^{1,11}$, su empleo por debajo de los 2-3 años debe realizarse con estricto cuidado ya que el cráneo no soporta la presión de los fijadores, existiendo riesgo de lesión ósea e intracaneal ${ }^{8}$. En la reciente revisión de Vitali y Steinbock ${ }^{12}$ sobre una población pediátrica de 766 niños intervenidos empleando un cabezal de Mayfiled, presentaron 5 casos $(0.65 \%)$ de lesión ósea craneal y hematoma epidural, estando la edad de los niños comprendida entre 2.7 y 6.6 años. En esta serie, en tres casos el diagnóstico de la fractura se realizó en el momento de aplicar el cabezal $\mathrm{y}$ en los otros dos casos el diagnóstico fue precedido de un deterioro neurológico del paciente. En la serie de Lee et $\mathrm{al}^{7}$, tienen 5 pacientes con complicaciones derivadas de los pinchos, aunque se trata de pacientes de más edad que los de Vitali y Steinbok ${ }^{12}$ entre 3 y 8 años. En cuatro casos la complicación fue detectada en el momento de colocar el 
cabezal antes de iniciar la cirugía: en 3 casos se detectó un hundimiento en el momento de cerrar el cabezal y en el otro caso se observó que el pincho penetraba en exceso dentro del cráneo. Los cuatro pacientes tuvieron que ser intervenidos para la corrección del hundimiento; en tres casos observaron lesión dural y cortical y en el niño restante, la fractura se diagnosticó en un control postoperatorio.

En pacientes adultos este tipo de complicaciones es poco habitual y en nuestra revisión únicamente hemos localizado 7 casos de complicaciones graves entre las que se incluyen casos de embolismo aéreo ${ }^{2,4,5}$, hematoma epidural ${ }^{13} \mathrm{y}$ aneurisma traumático de arteria temporal superficial ${ }^{6}$ (Tabla 1). Esta baja incidencia puede estar relacionada en nuestra opinión, con el empleo meticuloso y estricto del cabezal.

En nuestro paciente, se observó durante la segunda cirugía que presentaba un cráneo excesivamente fino para la edad del mismo, este adelgazamiento craneal estaba posiblemente en relación directa con la hipertensión intracraneal mantenida a lo largo de un período prolongado de tiempo.

\section{Bibliografía}

1. Aoki, N., Sakai, T.: Modified application of three-point skull clamp for infants. Neurosurgery 1989; 25: 660-662.

2. Cabezudo, J.M., Glsanz, F., Vaquero, J, Areitio, E., Martínez, R.: Air embolims from wounds from a pin-type head-holder as a complication of posterior fossa in the sitting position. J Neurosurg 1981; 55: 147-148.

3. Conde, C., Rumiá, J., García, G., Aguas, J., Gaston, F.: Tratamiento neuroendocópico de la hidrocefalia: nuevas posibilidades para una vieja técnica. Neurocirugía 1993; 3 : 196-204.

4. De Lange, J.J., Baerta, W.D., Booij, L.H.: Air embolism due to the Mayfield skull clamp. Acta Anaesthesiol Belg 1984; 35: 237-241.

5. Erbayraktar, S., Gökmen, N., Acar, Ü.: Intracranial penetrating injury associated with an intraoperative epidural haematoma caused by a spring-laden pin of a multipoise headrest. Br J Neurosurg 2001; 15: 425-428.
6. Fernández-Portales, I., Cabezudo, J.M., Lorenzana, L., Gómez, L., Porras, L., Rodríguez, J.A.: Traumatic aneurysm of the superficial temporal artery as a complicaton of pin-type head-holder device. Case report. Surg Neurol 1999; 52: 400403.

7. Lee, M., Rezai, A.R., Chou, J.: Depressed skull fractures in children secondary to skull clamp fixation devices. Pediatr Neurosurg 1992; 21: 174-178.

8. Márquez, J., Quiroga, E., Giménez, J., et al.: Astrocitomas supratentoriales. En: Villarejo F, Madero L eds. Tumores del sistema nervioso central en niños. Madrid: Ergos, 2007; pp 33-69.

9. Medina, M., Melcarne, A., Musso, C., Ettore, F.: Acute brain swelling during removal of supratentorial cystic lesion caused by contralateral extradural hematoma: case report. Surg Neurol 1997; 47: 428-431.

10. Sade, B., Mohr, G.: Depressed skull fracture and epidural hematoma: an unusual post-operatve complication of pin headrest in an adult. Acta Neurochir (Wien) 2005; 147 : 101-103.

11. Sgouros, S., Grainger, M.C., McCallin, S.: Adaptation of skull clamp for use in image-guided surgery of children in the first 2 years of life. Childs Nerv Syst 2005; 21: 101-103.

12. Vitali, A., M., Steinbok, P.: Depressed skull fracture and epidural hematoma from head fixation with pins for craniotomy in children. Childs Nerv Syst 2008; 24: 917-923.

13. Yan, H.,J.: Epidural hematoma following use of a three-point skull clamp. J Clin Neurosci 2007; 14: 691-693.

Serramito-García, R.; Arcos-Algaba, A.; Santín-Amo, J.M.; García-Allut, A.; Bandín-Diéguez, F.J.; GelabertGonzález, M.: Hematoma epidural secundario al empleo de cabezal autoestático en un adulto. Neurocirugía 2009; 20: $567-570$.

Correspondencia: Ramón Serramito García. Servicio de Neurocirugía. Hospital Clínico Universitario. La Choupana s/n. 15706 Santiago de Compostela ramonserramito@yahoo.es 